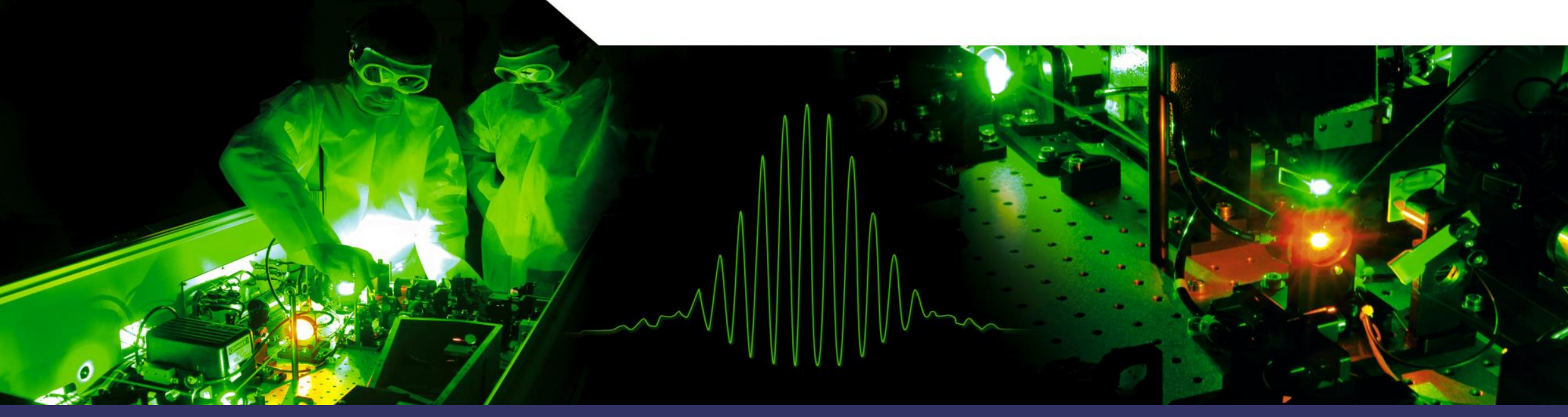

\title{
Improved conduction cooled compact laser for LIBS - Raman instruments
}

E. Durand, C. Derycke, A. Soujaeff, L. Boudjemaa, C. Simon-Boisson : Thales LAS France SAS

L. Roucayrol, M. Boutillier, B. Faure : CNES

S. Maurice : IRAP

ICSO 2018 - october $10^{\text {th }} 2018$ 


\section{- ChemCam laser}

- Context

- Optical configuration and performances

- SuperCam laser

- Context

- Optical configuration and performances

- Table top laser to increase laser performances

- Optical configuration

- Results

- Next generation of LIBS/RAMAN laser

- Conclusions 
- ChemCam is a French-American instrument aboard Curiosity rover on Mars since 08/2012

- Chemcam instrument made of 2 parts : "Mast Unit or MU" (IRAP/CNES) and "Body Unit" (LANL)

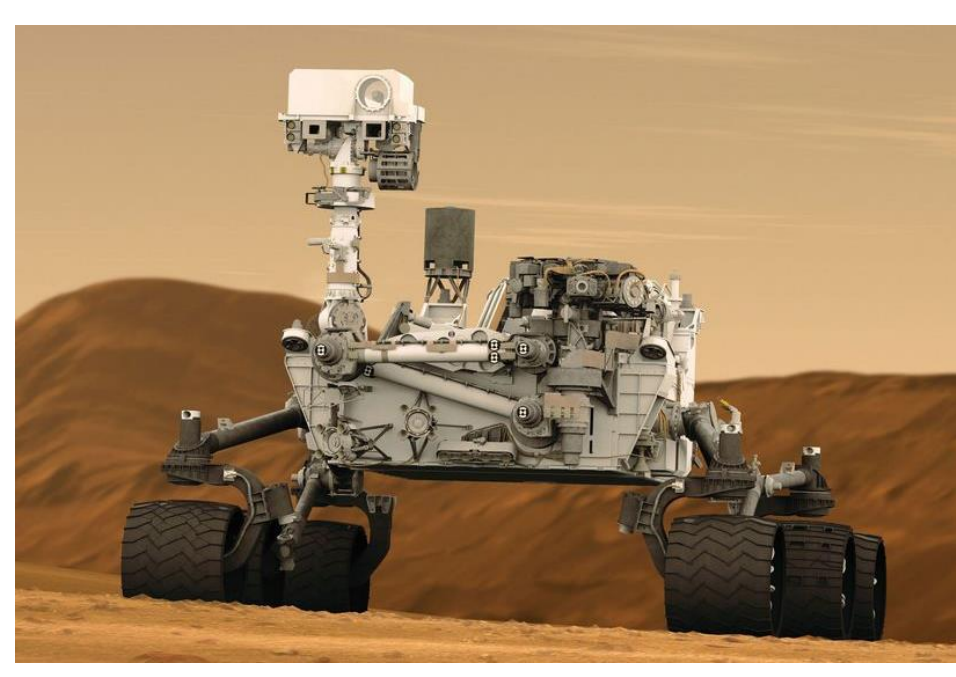

CNASA

- ChemCam instrument is performing LIBS analysis

- Thales in charge of the laser integrated in the MU

- ChemCam instrument has performed more than 600000 shots during its 6 years mission, still in progress 


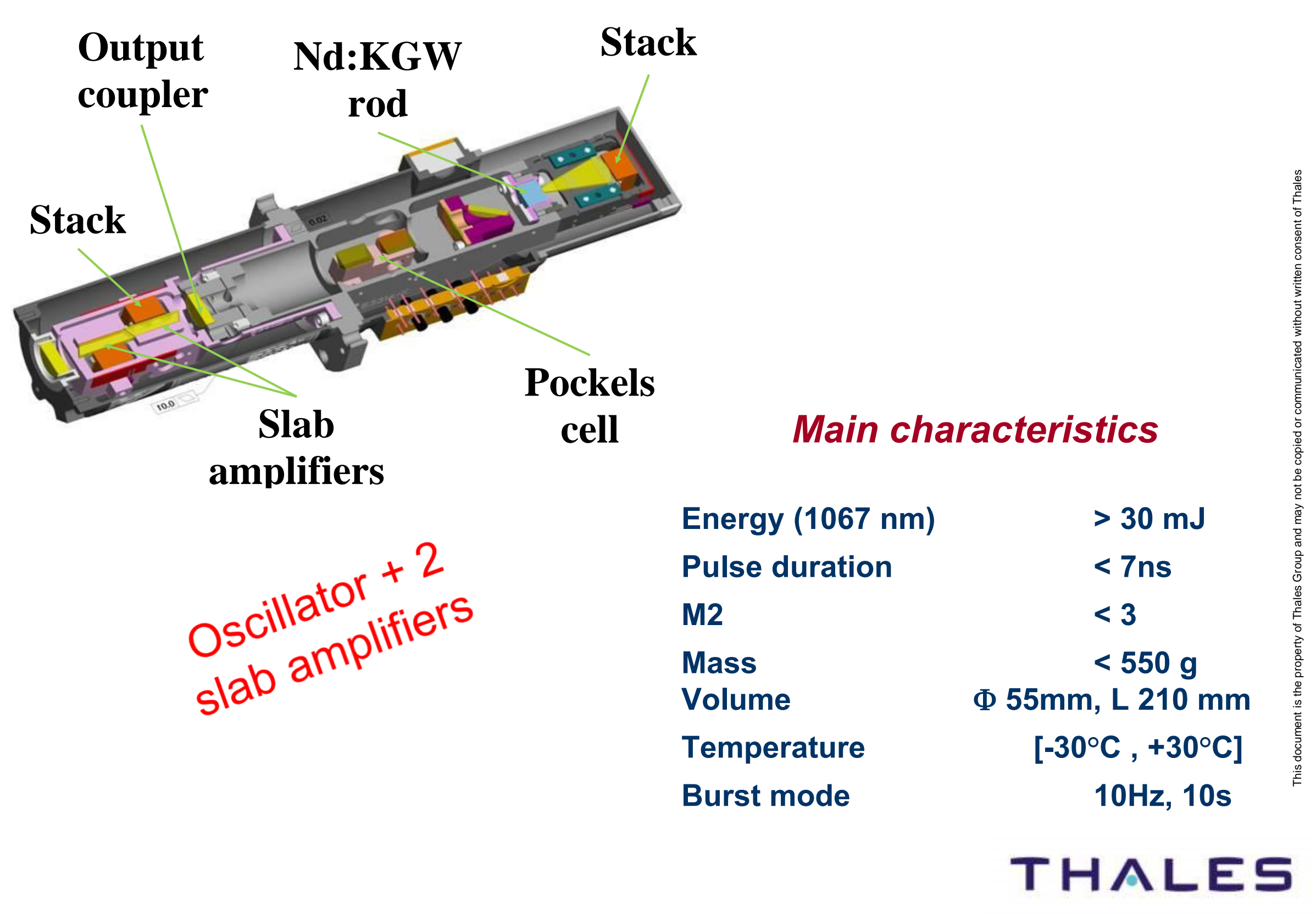




\section{SuperCam context}

- SuperCam a FrenchAmerican instrument aboard Mars 2020 rover to be launched in July 2020

- Main evolutions concern

- Raman analysis at 532 nm (new infrared spectrometer)

- Upgrade of Remote Micro Imager (RMI), which provides high-resolution context imaging (new color detector)

- Microphone added to detect sound waves during both SuperCam and Rover operations

- Thales in charge of the laser

- FM laser delivered to IRAP end of 2017

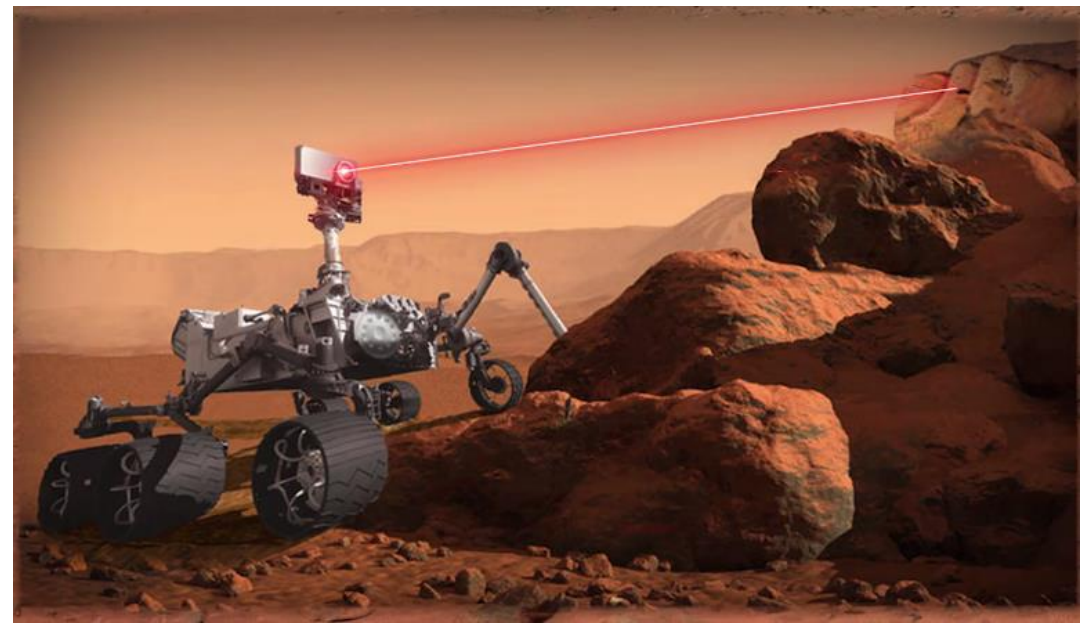

ONASA

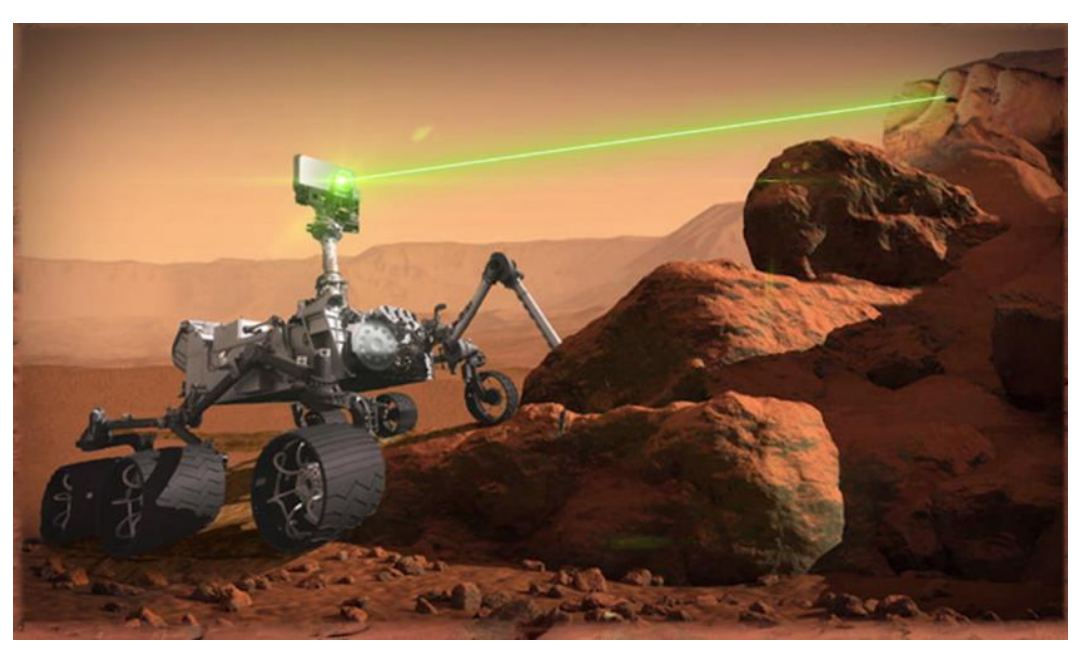

ONASA 
SuperCam laser : optical configuration and performances
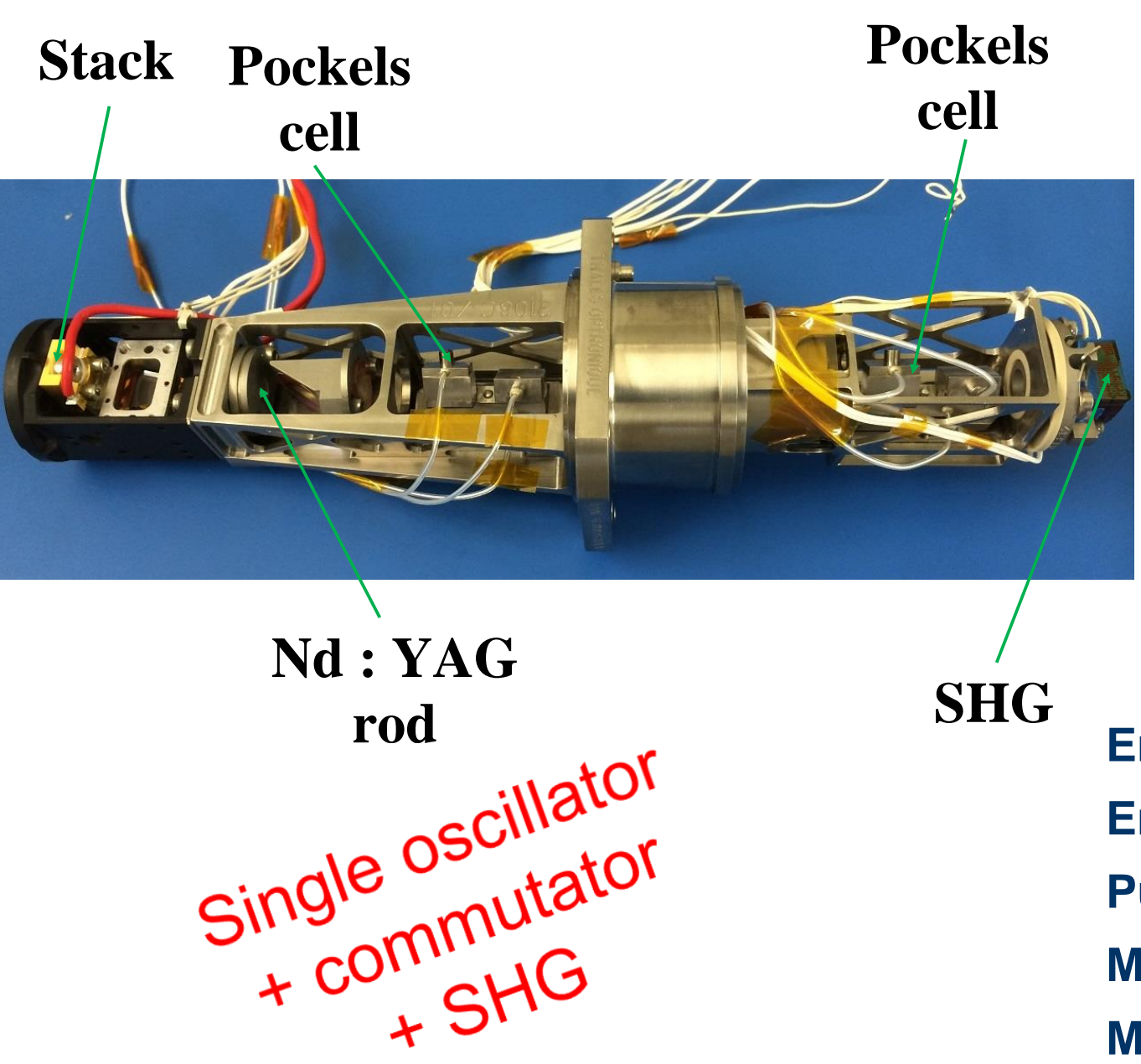

Design reported at ICSO 2016

\section{Main characteristics}

SHG

Energy (1064 nm)

Energy (532 nm)

Pulse Duration

M2

Mass

Volume

Temperature

Burst mode

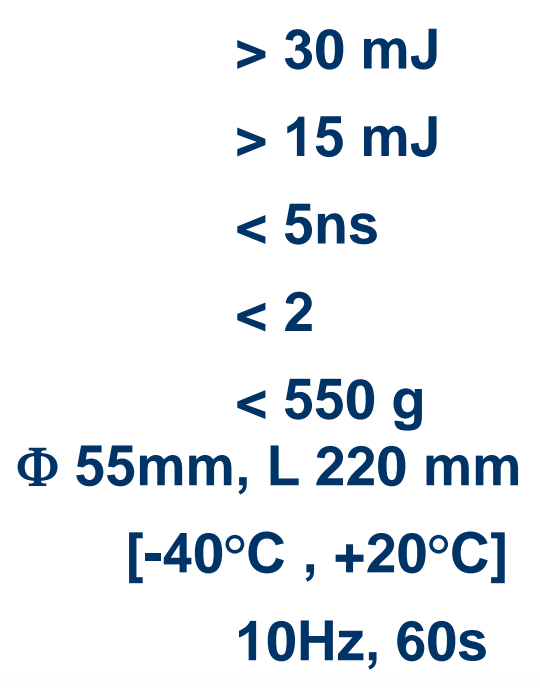$$
<2
$$$$
<550 \mathrm{~g}
$$

Ф 55mm, L 220 mm$$
\left[-40^{\circ} \mathrm{C},+20^{\circ} \mathrm{C}\right]
$$
$10 \mathrm{~Hz}, 60 \mathrm{~s}$ 
- Use space qualified building blocks on ChemCam and SuperCam lasers

- Higher energy with oscillator + amplifier design (implemented on ChemCam)

- Increase number of shots per burst using Nd .YAG material (SuperCam heritage)

- Raman capability using SHG (SuperCam heritage)

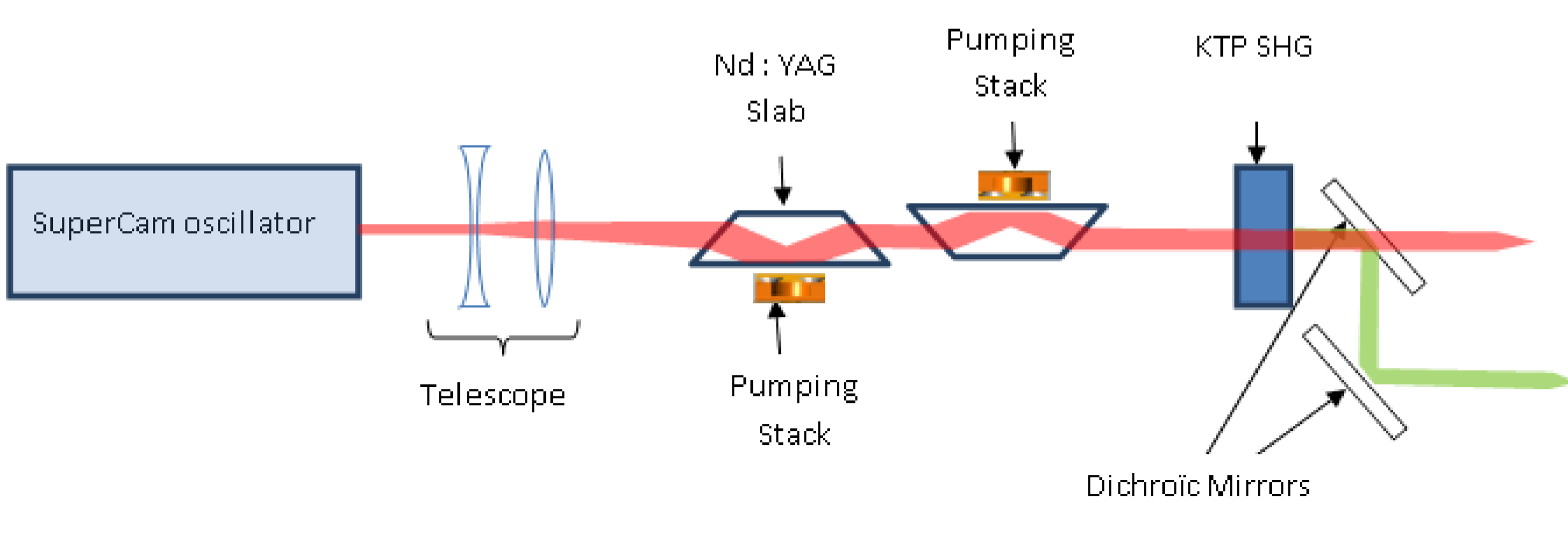




\section{Table top laser results}
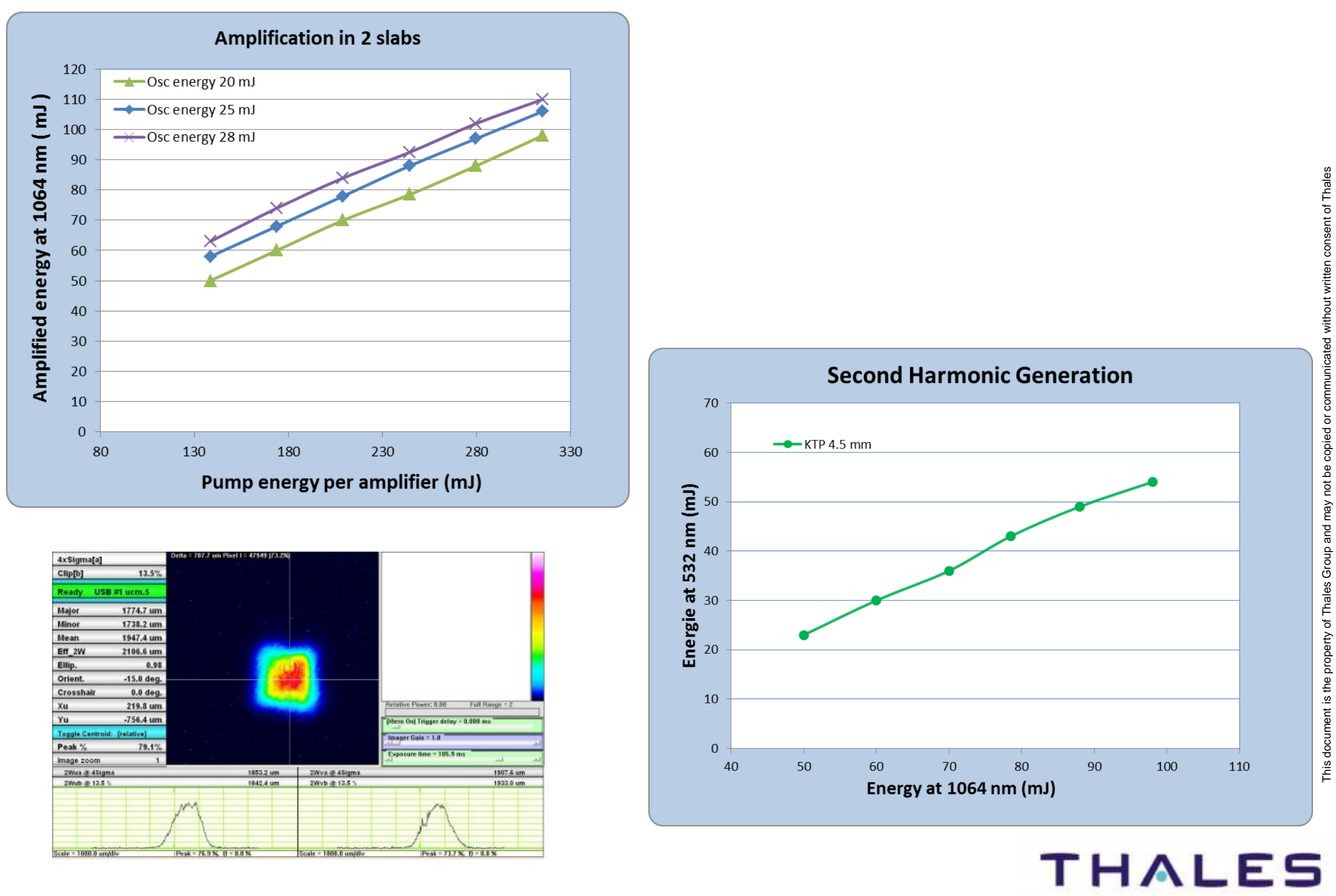
Stack

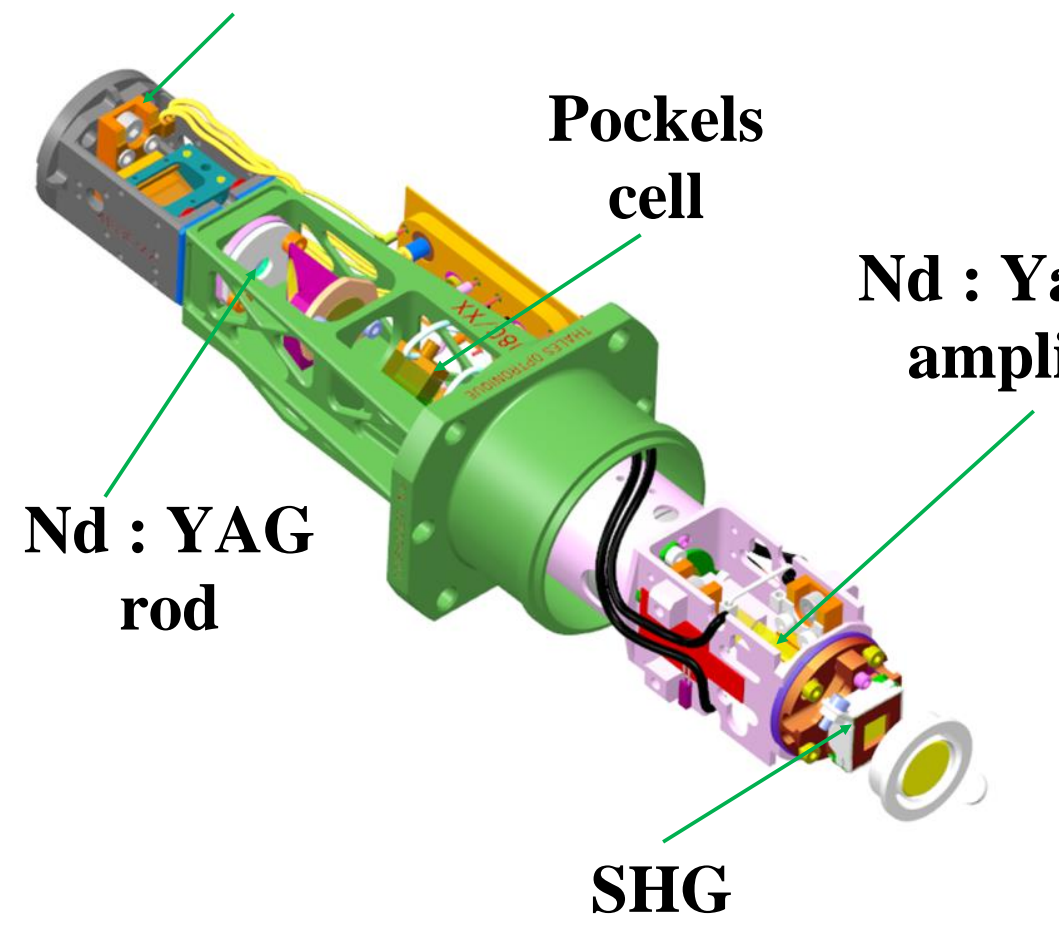

\section{Main characteristics}

Energy (1064 nm)
Energy (532 nm)
Pulse Duration
M2
Mass
Volume
Temperature
Burst mode

\begin{tabular}{|c|}
\hline$>100 \mathrm{~mJ}$ \\
\hline$>50 \mathrm{~mJ}$ \\
\hline$<5 n s$ \\
\hline$<2$ \\
\hline $\begin{array}{c}<550 \mathrm{~g} \\
\Phi 55 \mathrm{~mm}, \mathrm{~L} 220 \mathrm{~mm}\end{array}$ \\
\hline$\left[-40^{\circ} \mathrm{C},+20^{\circ} \mathrm{C}\right]$ \\
\hline $10 \mathrm{~Hz}, 60 \mathrm{~s}$ \\
\hline
\end{tabular}


Conclusions and perspectives

- Using SuperCam oscillator and two slab amplifiers (Chemcam heritage) pumped by multicolor stacks, a new laser has been built on a table top setup.

- 100 mJ output energy has been obtained at 1064 nm with good beam quality.

- Using a $4.5 \mathrm{~mm}$ KTP crystal, $50 \mathrm{~mJ}$ has been obtained at $532 \mathrm{~nm}$

- These results may be used to build the next generation of LIBS/RAMAN instrument in a volume not very different from SuperCam laser

Acknowledgement

This work was performed in the frame of CNES R\&T $n^{\circ}$ R-S17/SU-0003-057 